\title{
The LHCb Vertex Locator
}

\author{
Lars Eklund on the behalf of the LHCb VELO Group ${ }^{\text {a }}$ \\ ${ }^{\mathrm{a}}$ CERN, CH-1211 Genève 23, Switzerland
}

\begin{abstract}
LHCb is an experiment at the Large Hadron Collider (LHC), dedicated to the study of b-hadron physics. The Vertex Locator (VELO) is a sub-detector which reconstructs primary and secondary vertices, which is of importance for the processes under study. It is a silicon micro-strip detector operated in a harsh radiation environment with demands of high read-out speed and the use of minimal material. In this paper, the LHCb VELO is introduced and the technology choices of the detector are motivated. We report on the present status of the project and one possible upgrade technology is presented.
\end{abstract}

Key words: Silicon Micro-strip, Vertex Detector, Radiation Hardness PACS: 29.40.Gx

\section{Introduction}

The Large Hadron Collider (LHC) is a $14 \mathrm{TeV}$ proton-proton collider scheduled to be fully commissioned in 2007 at CERN. The LHCb experiment(1) is built at one of the interaction points of the LHC, to study the physics of b-flavoured hadrons. LHCb will make precision measurements of $\mathrm{CP}$ violating processes and hence constrain the elements of the CKM matrix. This is a sensitive probe for physics beyond the Standard Model. The LHCb experiment will operate at a luminosity of $10^{32} \mathrm{~cm}^{-2} \mathrm{~s}^{-1}$ which will generate in the order of $10^{12} \mathrm{~b} \overline{\mathrm{b}}$ pairs per year, with the full spectrum of b-hadrons.

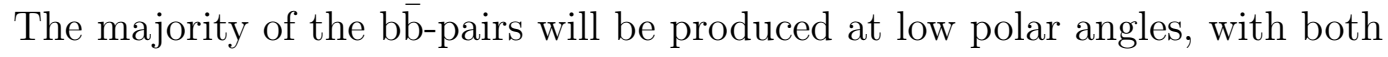
constituents of the correlated pair emitted in the same direction. For this reason, the LHCb experiment is built as a single forward spectrometer arm. A characteristic feature of b-flavoured hadrons is their relatively long lifetime, which is in the order of pico seconds. After being produced in the primary interaction vertex they subsequently decay and create a secondary vertex which is displaced from the primary vertex by a few $\mathrm{mm}$. The distance between 


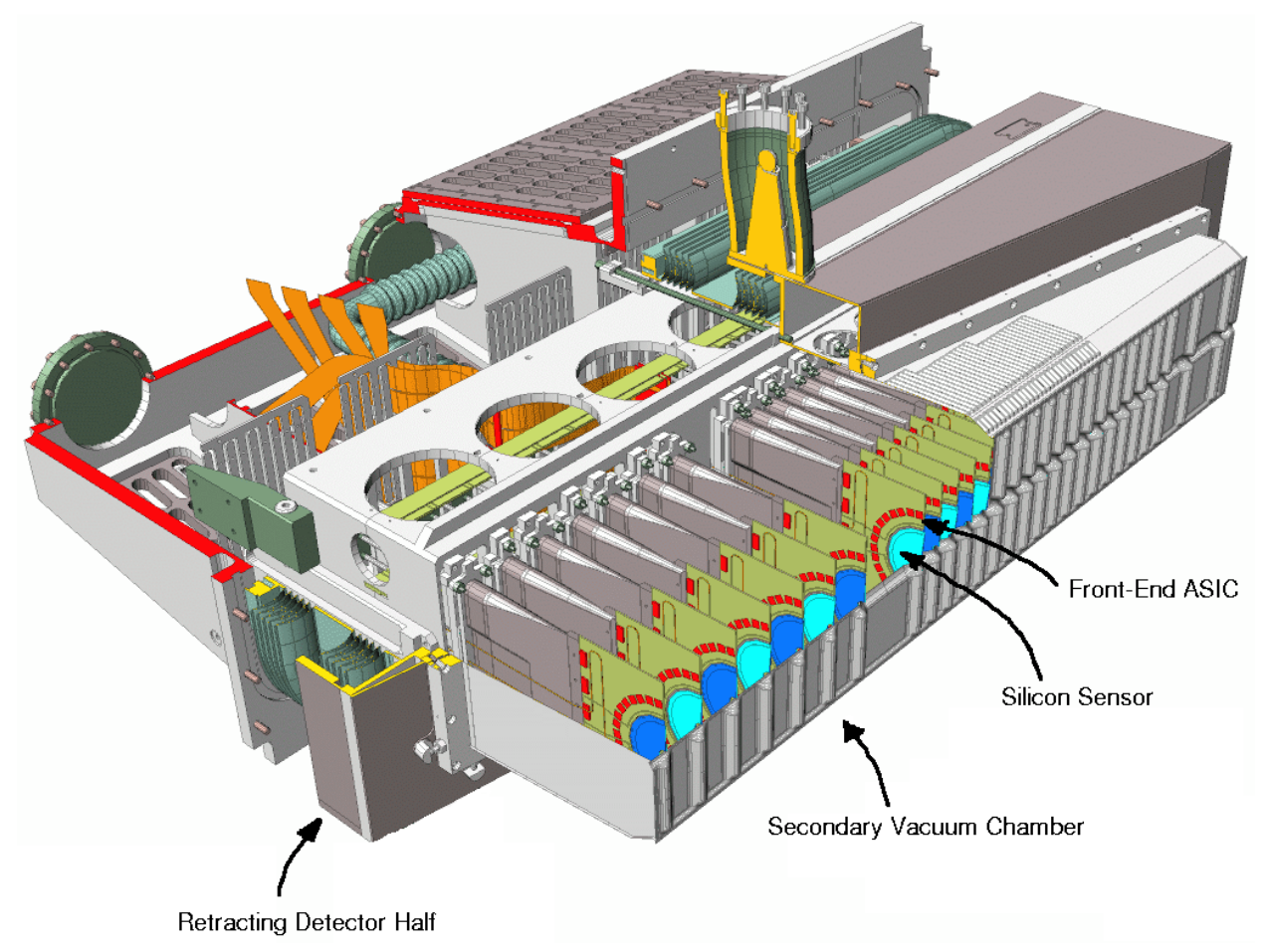

Fig. 1. Schematic picture showing one VELO detector half with the detector modules, the secondary vacuum chamber and the support mechanics.

the vertices is an important quantity to measure since it is a measure of the corresponding proper time. For instance, $\mathrm{LHCb}$ will measure fast $\mathrm{B}_{\mathrm{s}}$ oscillations with a proper time resolution of $40 \mathrm{fs}$. In addition, the decay length is a characteristic used to identify b-flavoured hadrons which is used in the event selection of the second and higher level triggers.

\section{The Vertex Locator}

The $\operatorname{VELO}(2)$ is the sub-detector in $\mathrm{LHCb}$ which reconstructs the primary and secondary vertices. It has 21 tracking stations positioned orthogonally to the beam, around and downstream from the interaction point. Each tracking station has two detector modules, mounted on separate retracting detector supports (see Fig. 1). This enables the retraction of the two detector halves during the set-up of the colliding beams. The first sensitive element, in the closed position, is $8.2 \mathrm{~mm}$ from the beam-line. This short distance reduces the extrapolation error in the determination of the vertex position. To reduce the material before the first measured point the VELO is operated in a secondary vacuum, separated from the primary machine vacuum only by a $250 \mu \mathrm{m}$ thick aluminium foil. 
The double sided detector modules consist of two silicon sensors mounted back-to-back with different micro-strip geometry. The R-sensor measures the radial distance from the beam axis and the $\Phi$-sensor measures an approximate azimuthal angle $\Phi$, where the radial strips have a stereo angle to give an additional measurement of the radial position. The pitch on the R-sensor varies from 40 to $102 \mu \mathrm{m}$ and on the $\Phi$-sensor from 36 to $97 \mu \mathrm{m}$, with the smallest pitch at low radii. This geometry gives the computational efficiency needed for the fast track reconstruction of the second level trigger decision. In addition it spreads the occupancy evenly across the channels and gives the highest precision to the first measured point. The bunch-crossing rate of $40 \mathrm{MHz}$ requires the signal shaping time to be less than 25 ns. The signals are routed out via a second metal layer to an analogue front-end ASIC. The sensors and ASICs are mounted on a TPG (Thermalised Pyrolytic Graphite) and carbon-fibre sandwich, with a kapton flex circuits laminated on each side.

\section{Technology Choices}

The VELO will operate in a harsh non-uniform radiation environment which has been one of the leading design criteria. The maximal expected fluence is $1.3 * 10^{14} \mathrm{~cm}^{-2} 1 \mathrm{MeV}$ neutrons NIEL equivalent per annum for the innermost

radius of the tracking station closest to the interaction point. The fluence decreases roughly as $\mathrm{r}^{-2}$ with increasing radius $\mathrm{r}$ away from the beam axis. The VELO uses Diffusion Oxygenated Float Zone (DOFZ) micro-strip silicon sensors. Float Zone (FZ) silicon is at present the standard of wafer fabrication for particle physics applications. To further increase the radiation hardness, the VELO uses oxygen enriched silicon sensors. It has been shown(3) that an enhanced level of oxygen $\left(10^{17} \mathrm{~cm}^{-3}\right)$ in the silicon bulk slows down the change of effective doping concentration due to radiation damage.

Towards the end of the lifetime of the VELO, the sensors may be operated partially under-depleted. Therefore, the sensors are made as $\mathrm{n}^{+}$strips in $\mathrm{n}$-bulk, with a $\mathrm{p}^{+}$ohmic contact in the back-plane. Hence after type-inversion the sensors will depleted from the strip-side and there will be no loss in resolution and only a limited loss in charge collection if operated at partial under-depletion. This was demonstrated in a beam test with a double sided n-bulk detector, comparing performance of the sides with $\mathrm{n}^{+}$and $\mathrm{p}^{+}$strips(4).

\section{Present Status}

The VELO project is now in the final stages of R\&D, verifying the performance of the silicon sensors and the front-end electronics. Close to final prototypes 


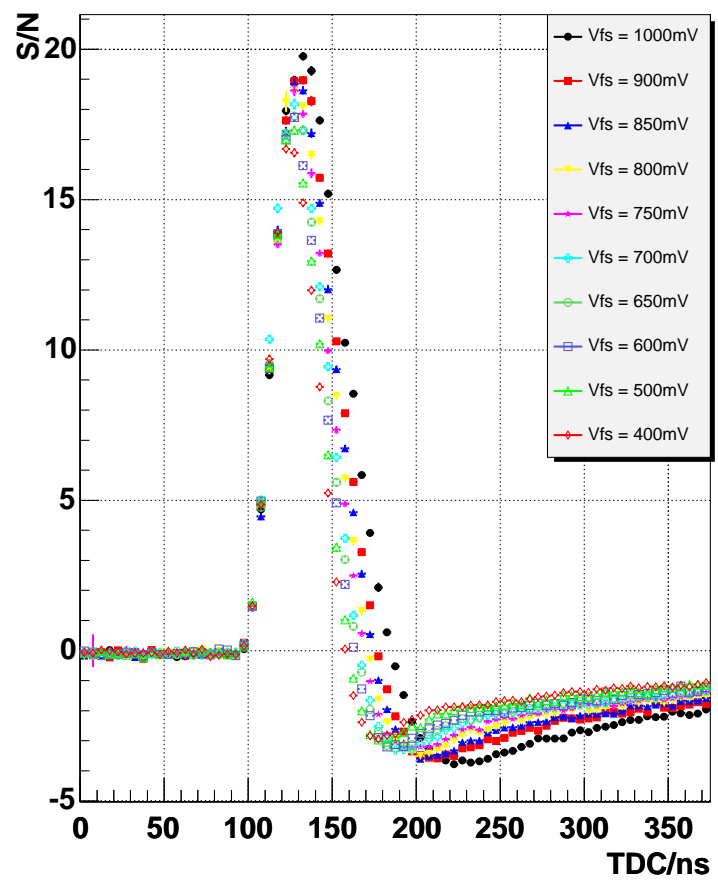

Fig. 2. Front-end pulse shapes for different bias voltages $\left(\mathrm{V}_{\mathrm{fs}}\right)$ on the gate of the MOS transistor across the shaper feed-back capacitor. Results from beam test at CERN, June 2004.

have been evaluated in beam tests and test bench measurements(5) via a prototype of the analogue data link. Analysis and optimisation procedures are in progress. Two competing criteria of the front-end performance are sufficient signal to noise ratio while maintaining a acceptable signal remainder after 25 ns. In 2004 a beam test was performed which scanned the front-end ASIC settings while reconstructing the analogue pulse shapes. As an example, Fig. 2 shows the pulse shapes for different settings of the parameter $\mathrm{V}_{\mathrm{fs}}$. Moreover, algorithms for data processing are being developed and optimised before implementation in the experiments final firm- and software.

In parallel with the $\mathrm{R} \& \mathrm{D}$ activities, procedures and tooling for production and assembly are prepared. This includes the production and validation of the detector modules themselves as well as the integration of the modules into a complete detector system.

\section{$5 \quad$ VELO Upgrades}

The design criterion for the radiation hardness of the silicon sensors is three years of operation in $\mathrm{LHCb}$. The LHC machine is foreseen to operate for ten 
years, implying that the VELO detector modules will require replacement. Several different scenarios for an upgraded VELO are under study, making use of recent technological advances. Several sensor technologies are studied, e.g. 3D detectors, n-in-p sensors and pixels. In this paper, we report on a study of Magnetic Czochralski silicon (MCz)(6). Czochralski silicon has until recently not reached sufficient purity for use in particle detectors, even though it has been the standard crystal growth process for the electronics industry. $\mathrm{MCz}$ silicon is now available with high resistivity and is due to its natural high oxygen content $\left(10^{18} \mathrm{~cm}^{-3}\right)$ expected to be very radiation hard.

To evaluate $\mathrm{MCz}$ as a material for a VELO upgrade, a beam test with a full scale $\mathrm{MCz}$ micro-strip sensor read out at LHC speed was performed. The $\mathrm{MCz}$ sensor was irradiated with $24 \mathrm{GeV}$ protons to a non-uniform fluence with a peak value of $7 * 10^{14} \mathrm{~cm}^{-2}$. The sensor was measured in a beam before and after irradiation, hence the performance could be evaluated for a range of fluencies(7). The signal to noise for the $380 \mu \mathrm{m}$ thick sensor was 23.5 before irradiation and 11 after a fluence corresponding to two years of VELO operation. However, the sensor was operated under-depleted after irradiation and the analysis was limited by statistics so it was not possible to explore the full potential of the material. Studies to evaluate $\mathrm{MCz}$ silicon and other upgrade options will continue in the years to come.

\section{References}

[1] LHCb Technical Design Report, Reoptimized Detector Design and Performance, CERN/LHCC, 2003-030 (2003)

[2] LHCb VELO Technical Design Report, CERN/LHCC 2001-011 (2001)

[3] G. Lidström et al., "Radiation hard silicon detectors - developments by RD48 (ROSE) collaboration," Nucl. Instrum. Meth. A 466 (2001) 308-326.

[4] K. Borer et al., "Charge collection efficiency and resolution of an irradiated double-sided silicon micro-strip detector operated at cryogenic temperatures," Nucl. Instrum. Meth. A 440 (2000) 17-37.

[5] A. Bates et al., "Pulseshape characteristics of a $300 \mu \mathrm{m}$ PR03 sensor read out through a Beetle1.3 chip", LHCb Collaboration Note, LHCB 2004-068 VELO

[6] V. Savolainen et al., "Simulation of large-scale silicon melt flow in magnetic Czochralski growth", J. Crystal. Growth 243 (2003), 2.

[7] A. Bates et al., "Results from the first test beam of a large microstrip Czochralski silicon detector equipped with LHC speed electronics", LHCb Collaboration Note, LHCB 2004-52 VELO 